\title{
Novelty effects on recollection and familiarity in recognition memory
}

\author{
MARK M. KISHIYAMA and ANDREW P. YONELINAS \\ University of California, Davis, California
}

\begin{abstract}
Recognition memory is better for novel or distinctive items than for non-novel items. However, it is not known whether these effects reflect changes in recollection or in familiarity-based recognition judgments. Some previous results have indicated that recollection should be more sensitive to novelty than to familiarity, whereas other results have suggested the opposite. We used a von Restorff paradigm in which a small proportion of studied items were made novel by presenting them in a color different from that of the majority of the study items. Memory was tested using a remember-know procedure. Across two experiments, stimulus novelty was found to benefit both recollection and familiarity. The effects on familiarity were observed under intentional and incidental encoding conditions, whereas the effects on recollection were significantly reduced, and no longer significant, under incidental as compared with intentional encoding conditions. Thus, both processes benefit from stimulus novelty, but the extent to which recollection benefits from novelty depends on the encoding condition.
\end{abstract}

The ability to detect novelty is a fundamental characteristic of all mammalian nervous systems (Sokolov, 1963), and it plays a critical role in memory in the sense that items that are novel, or distinctive, are remembered better than those that are less distinct (e.g., Hunt, 1995; Kinsbourne \& George, 1974; Parker, Wilding, \& Ackerman, 1998; Saltz \& Newman, 1959; Tulving \& Kroll, 1995; von Restorff, 1933; see Wallace, 1965, for a review). For example, in a paradigm developed by von Restorff, subjects are exposed to a list of items that includes a subset of items that are made novel or distinctive from the majority. Subsequently, subjects are found to exhibit enhanced recall for novel, as compared with non-novel, items (Hunt, 1995; Saltz \& Newman, 1959; von Restorff, 1933; Wallace, 1965). This effect is extremely robust and has been observed when items are made novel on a variety of stimulus dimensions, such as color, size, brightness, and semantic category (e.g., Cimbalo, 1978; Hunt, 1995; Wallace, 1965).

Although the effects of novelty on free recall have been studied quite extensively, relatively little is known about how novelty influences the processes supporting recognition memory. Results from behavioral, neuroimaging, and patient studies, as well as from studies of rats and nonhuman primates, have indicated that recognition memory judgments can be supported by recollection of qualitative information about previous study events or by assessments of stimulus familiarity (for reviews, see Aggleton \& Brown, 1999; Yonelinas, 2002).

Correspondence concerning this article should be addressed to M. Kishiyama, Department of Psychology, One Shields Avenue, University of California, Davis, CA 95616 (e-mail: mmkishiyama@ ucdavis.edu).
Although novelty has been found to influence overall recognition memory performance (e.g., Fabiani \& Donchin, 1995; Parker et al., 1998; von Restorff, 1933), we currently do not know whether novelty influences recollection or familiarity-based recognition judgments.

Determining how novelty influences recollection and familiarity promises to provide an important way of differentiating between these two processes. For example, Rajaram $(1996,1998)$ has suggested that the analysis of salient or distinctive aspects of items leads to recognition memory responses accompanied by the subjective experience of remembering, whereas variations in the processing fluency of items gives rise to feelings of familiarity in the absence of recollection. Although Rajaram $(1996,1998)$ did not make any claims about recollection and familiarity processes per se but, rather, focused exclusively on the subjective experiences of remembering and knowing, it is possible that this distinction also holds for the processes of recollection and familiarity that give rise to these subjective experiences. We will refer to this as the recollection/distinctiveness hypothesis.

Support for the recollection/distinctiveness hypothesis comes from two sources. First, because robust novelty effects have been observed in free recall (e.g., Hunt, 1995; Saltz \& Newman, 1959; von Restorff, 1933; Wallace, 1965) and recollection is expected to be functionally similar to the search process involved in free recall (Mandler, 1980), it follows that recollection should be sensitive to novelty effects. Second, Rajaram (1998) found that perceptually distinctive words were found to facilitate recollection-based recognition judgments but not familiarity-based recognition responses. In that experiment, subjects studied a list containing orthographically distinct words (e.g., subpoena) and orthographically common words (e.g., sailboat). Recognitionmemory was then 
tested using the remember-know procedure (Gardiner, 1988; Gardiner \& Java, 1991; Gardiner \& Parkin, 1990; Tulving, 1985), in which subjects indicated whether they recollected some qualitative information about the study event (i.e., a remember response), recognized the word on the basis that it was familiar in the absence of recollection (i.e., a know response), or thought the word was new. The results indicated that orthographically distinctive words led to an increase in reports of remembering, as compared with common words, but did not influence the level of know responses, suggesting that recollection is more sensitive to stimulus novelty than is familiarity.

There is, however, reason to question the recollection/ distinctiveness hypothesis and to suspect that it is familiarity, rather than recollection, that is particularly sensitive to novelty. We will refer to this as the familiarity/novelty hypothesis. The motivation for this hypothesis is that novelty and familiarity might reflect two extremes of a continuum. That is, it seems quite parsimonious to assume that the same mechanism that causes an item to appear novel might also be used as a basis for familiaritybased recognition judgments (e.g., "if it appears to be novel and I don't remember studying the item, then it probably was not studied").

The familiarity/novelty hypothesis is supported by Parker et al.'s (1998) study, in which novelty was examined using the von Restorff paradigm. In their study, human and nonhuman primates exhibited better recognition memory for novel items (i.e., visual shapes presented in a novel color, as compared with shapes presented in a color that was more common in the experiment). The study did not include separate measures of recollection and familiarity. However, lesions in the nonhuman primates designed to isolate the hippocampal formation were not found to disrupt the novelty effects, whereas lesions that included the surrounding parahippocampal gyrus eliminated the novelty effects. In light of previous studies that have indicated that the hippocampus supports recollection, whereas the surrounding parahippocampal gyrus is involved in supporting familiarity-based recognition responses (e.g., Aggleton \& Brown, 1999; Eichenbaum, Otto, \& Cohen, 1994; Yonelinas, 2002; Yonelinas et al., 2002), these results indicate that stimulus novelty influences familiarity-based recognition but not recollection-based recognition.

To test the recollection/distinctiveness and familiarity/ novelty hypotheses, the present study included two experiments in which the effects of novelty on recollection and familiarity-based recognition were examined. A von Restorff procedure was used in which a study list was presented that contained a majority of non-novel items (e.g., black thumbnail object images) mixed with a small number of novel items (e.g., colored thumbnail object images). The color manipulation was used because previous studies of the von Restorff effect have indicated that it is one of the most effective techniques for producing novelty effects (Cimbalo, Capria, Neider, \& Wilkins, 1977). Subjects were then presented with a list of studied and new items and were required to make recognition judgments. For each test item, the subjects were required to make a remember response if they could recollect some qualitative information about the study event in which that item had earlier been presented, a know response if the item was familiar but they could not recollect anything specific about the study event, or a new response if they thought the item had not been studied (Gardiner, 1988; Tulving, 1985). The remember and know responses were used to assess recollection and familiaritybased recognition.

In Experiment 1, we examined recognition memory after intentional encoding conditions (as have most previous studies of the von Restorff effect). Because the results of the first experiment were unexpected-novelty led to increases in both recollection and familiaritybased recognition-Experiment 2 was conducted to test the replicability of the initial findings and to assess the generalizability of those results across different encoding conditions (i.e., intentional and incidental encoding).

\section{EXPERIMENT 1}

\section{Method}

Subjects and Materials. Sixty undergraduates at the University of California, Davis received course credit for their participation. A total of 660 thumbnail object images (e.g., animals, tools, symbols, etc.) from MasterClips Premium Image Collection (MasterClips, 1998) served as study and test items.

Procedure. The subjects were told that a long series of objects would be presented on the computer monitor at a rapid rate and that they were to try to remember them for a later memory test. They were told that some of the objects would appear in color and that others would appear in black (all the objects were displayed against a white background). They were told that color was not important and that they were to try to remember all the objects.

During the study phase, the subjects were presented with 600 items at a rate of $500 \mathrm{msec}$ per item. A long list was used in order to maximize the number of novel items while maintaining a low ratio of novel to non-novel items (i.e., one novel for every 20 items; Fabiani \& Donchin, 1995), and the fast presentation rate was used to avoid ceiling effects. Within the study list, there were 60 critical study items; 30 were novel in the sense that they were presented in a color (i.e., an equal number were presented in red, green, blue, yellow, cyan, and purple), and 30 were non-novel (i.e., they were presented in black). There were also 540 filler items (black objects). The items were presented in a pseudorandom order in such a way that novel objects were separated by a minimum of 10 intervening black objects. Novel and non-novel items were counterbalanced across subjects in the study list.

At test, the subjects were presented with 120 items, one at a time: 30 old and 30 new novel items (i.e., colored items), mixed with 30 old and 30 new non-novel items (i.e., black items). Both old and new novel and non-novel items were counterbalanced across subjects in the test list. The test items appeared in the same color as that in the study phase, because Fabiani and Donchin (1995) had found that it was necessary to reinstate the novel feature at test to obtain robust von Restorff effects in recognition memory.

For each item, the subjects were required to make a remember, a know, or a new judgment by pressing designated keys on a computer keyboard. The remember-know instructions were adapted from Gardiner (1988). The subjects were told that they were to respond remember if they could recollect some qualitative informa- 
Table 1

The Probabilities of Remember (R) and Know (K) Responses in Experiments 1 and 2 (With Standard Deviations)

\begin{tabular}{|c|c|c|c|c|c|c|c|c|}
\hline \multirow[b]{3}{*}{ Condition } & \multicolumn{4}{|c|}{ Old Items } & \multicolumn{4}{|c|}{ New Items } \\
\hline & \multicolumn{2}{|c|}{$\mathrm{R}$} & \multicolumn{2}{|c|}{$\mathrm{K}$} & \multicolumn{2}{|c|}{$\mathrm{R}$} & \multicolumn{2}{|c|}{$\mathrm{K}$} \\
\hline & $M$ & $\overline{S D}$ & $M$ & $\overline{S D}$ & $\bar{M}$ & $\overline{S D}$ & $\bar{M}$ & $\overline{S D}$ \\
\hline \multicolumn{9}{|c|}{ Experiment 1} \\
\hline Novel & .38 & .19 & .26 & .16 & .07 & .08 & .16 & .13 \\
\hline Non-novel & .36 & .18 & .25 & .13 & .10 & .11 & .24 & .18 \\
\hline \multicolumn{9}{|c|}{ Experiment 2} \\
\hline \multicolumn{9}{|l|}{ Intentional } \\
\hline Novel & .38 & .15 & .29 & .16 & .02 & .03 & .09 & .07 \\
\hline Non-novel & .33 & .19 & .34 & .15 & .06 & .10 & .23 & .17 \\
\hline \multicolumn{9}{|l|}{ Incidental } \\
\hline Novel & .42 & .14 & .21 & .09 & .10 & .06 & .19 & .16 \\
\hline Non-novel & .42 & .14 & .19 & .14 & .11 & .07 & .24 & .14 \\
\hline
\end{tabular}

tion about the study event. Moreover, they were instructed that they should respond remember only if they could, if asked, tell the experimenter what they recollected about that study event. The subjects were told to respond know if they thought the item had been studied but they could not recollect any details about the study event. That is, they should respond know if the item was familiar but not recollected. They were told to respond new if they thought the item was not in the study list. To ensure that the subjects understood the test instructions, they were asked to describe the remember-know distinction back to the experimenter, and the instructions were repeated if the subject appeared to misunderstand the distinction.

\section{Results and Discussion}

Table 1 presents the mean proportion of remember and know responses for novel and non-novel items. The remember and know responses were used to derive estimates of recollection and familiarity. Because the subjects were instructed to respond remember whenever an item was recollected, the probability of a remember response was used as an estimate of recollection (i.e., $R=$ remember). To incorporate false alarm rates, accuracy was measured using $d$, so that the proportions of correct and incorrect remember responses were used as hits and false alarms (MacMillan \& Creelman, 1991). Because the subjects were instructed to respond know when an item was familiar in the absence of recollection [i.e., know $=F(1-R)$ ], familiarity was estimated as the probability of a know response given that an item was not recollected [i.e., $F=$ know $(1-R)$; Yonelinas \& Jacoby, 1995]. The estimates of familiarity for old and new items were used as hits and false alarms to derive $d$ ' estimates of familiarity accuracy. Note that previous studies have indicated that recollection is most appropriately measured using probabilities (e.g., hits minus false alarms), whereas familiarity is most appropriately measured using the $d$ index from signal detection theory (Yonelinas, 2001). To facilitate direct comparisons of recollection and familiarity, we measured both processes by using the $d$ 'index. However, analyses of recollection and familiarity estimates, using probabilities as accuracy measures, led to the same pattern of results.
Figure 1 shows the estimates of recollection and familiarity based on the remember and know responses. Recollection was greater for novel than for non-novel items $[t(59)=2.89, p<.01]$. Moreover, familiarity estimates were also found to be higher for novel than for non-novel items $[t(59)=5.36, p<.01]$. Thus, the results indicate that both recollection and familiarity increased with stimulus novelty.

\section{EXPERIMENT 2}

The results of Experiment 1 do not provide particularly strong support for either the recollection/distinctiveness or the familiarity/novelty hypothesis. The recollection/ distinctiveness hypothesis leads to the expectation that only recollection should be sensitive to novelty. This clearly was not the case. In fact, a direct comparison of the novelty effects in Experiment 1 indicated that the effects on familiarity were significantly greater than those seen on recollection $[t(59)=2.39, p<.05]$. The finding that familiarity seemed to be more sensitive to novelty than was recollection is in partial agreement with the familiarity/novelty hypothesis; however, the expectation based on Parker et al.'s (1998) study was that recollection should be unaffected by the novelty manipulation, and this was not the case.

Given the apparent inconsistency of the initial results with previous studies, we decided to conduct a second experiment to determine whether the results were replicable. To further test the generalizability of the findings, we examined performance under two different encoding conditions: intentional and incidental encoding. Previous studies of free recall have shown that the von Restorff effect is reduced under conditions of incidental encoding (Gleitman \& Gillett, 1957; Postman \& Phillips, 1954; Saltzman \& Carterette, 1959) but not of intentional encoding (Koyanagi, 1957). Thus, one aim of Experiment 2 was to determine whether the results seen in free recall would generalize to recognition in such a way that novelty effects would be reduced under incidental, 


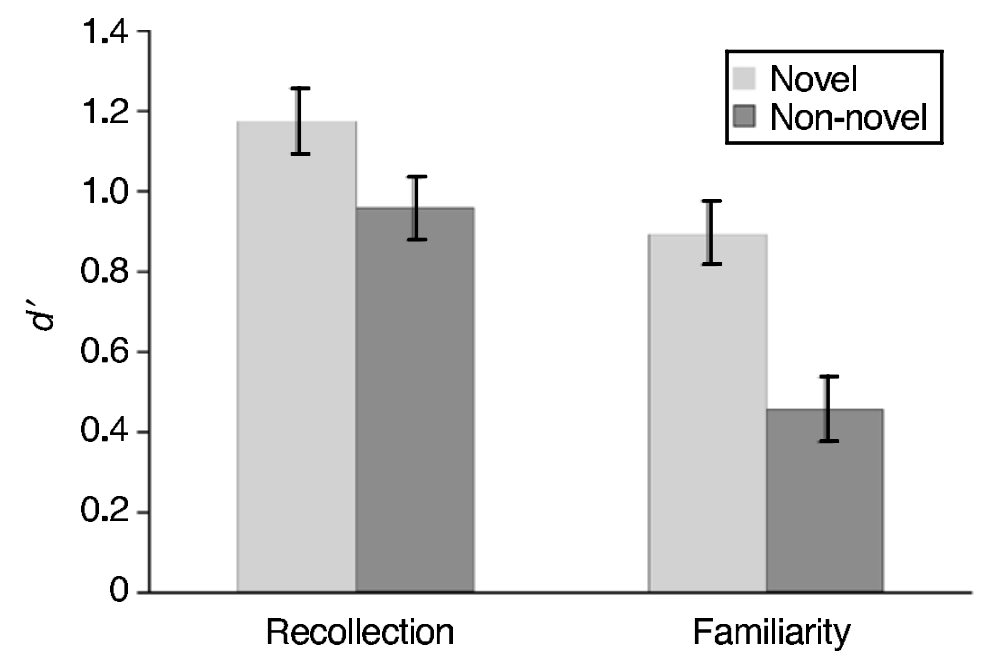

Figure 1. Experiment 1: Estimates of recollection and familiarity for novel and non-novel items.

relative to intentional, encoding conditions. If recollection involves a retrieval process that is similar to that involved in recall (e.g., Mandler, 1980), one would expect the effects of novelty on recollection to be reduced. Whether the effects of novelty on familiarity would also be influenced was not clear.

\section{Method}

Subjects and Materials. Forty undergraduates at the University of California, Davis received course credit for their participation. Half of the subjects were randomly assigned to the intentional encoding group, and half were assigned to the incidental encoding group. The materials were the same as those in Experiment 1, except that all the objects appeared in one of two different colors (e.g., red stimuli with a white background or yellow stimuli with a black background), rather than in color and black. The stimuli and colors were counterbalanced across subjects.

Procedure. The procedure was similar to that used in Experiment 1 , with the following exceptions. The subjects in the intentional group were told to try to remember the study items for a later memory test (as in Experiment 1). In contrast, the subjects in the incidental group were not told that their memory would be tested; rather, they were instructed to count the number of symmetrical objects they saw in the list. A symmetrical object was described to the subjects as an object that would have an identical form on either side if it were to be split down its vertical axis. To allow for sufficient time to complete the symmetry judgments, the presentation duration during the study phase was increased from 500 to $850 \mathrm{msec}$ per item for both conditions. The symmetry task was selected because it led to performance levels that were roughly comparable to those in the intentional condition. A test for colorblindness was administered after the test phase for all the subjects (Ishihara, 1998) and indicated that none of the subjects was colorblind.

\section{Results and Discussion}

Table 1 presents the mean proportions of remember and know responses for novel and non-novel items, and Figure 2 presents the estimates of recollection and familiarity. These estimates were derived from remember and know responses, using the same procedures as those described in Experiment 1.
Recollection was greater for novel than for non-novel items $\left[F(1,38)=6.83, M S_{\mathrm{e}}=0.114, p<.05\right]$, indicating that novelty improved recollection. There was no overall difference in recollection levels between the intentional and the incidental groups $\left[F(1,38)=0.75, M S_{\mathrm{e}}=0.360\right.$, $p=.39]$, but there was a significant item $\times$ group interaction $\left[F(1,38)=4.74, M S_{\mathrm{e}}=0.114, p<.05\right]$, which reflected the fact that the novelty effect on recollection was significantly larger under intentional than under incidental encoding conditions. Moreover, direct comparisons indicated that recollection estimates for the intentional group were greater for novel than for non-novel items $[t(19)=2.94, p<.01]$, whereas there was no significant difference between novel and non-novel items in the incidental condition $[t(19)=0.38, p=.70]$.

Familiarity estimates were greater for novel than for non-novel items $\left[F(1,38)=15.02, M S_{\mathrm{e}}=0.215, p<.01\right]$, indicating that novelty increased the familiarity estimates. Moreover, familiarity was greater for the intentional than for the incidental group $[F(1,38)=13.76$, $\left.M S_{\mathrm{e}}=0.532, p<.01\right]$, but the item $\times$ group interaction was not significant $\left[F(1,38)=0.21, M S_{\mathrm{e}}=0.215, p=.65\right]$, indicating that comparable novelty effects on familiarity were observed in the intentional and the incidental conditions. The novelty effects on familiarity were significant for the incidental $[t(19)=2.10, p<.05]$ and the intentional $[t(19)=3.73, p<.01]$ encoding conditions.

In sum, the results of the experiment replicate those in Experiment 1, showing that under intentional encoding conditions, both recollection and familiarity increased with stimulus novelty. They extend those findings, however, by showing that the novelty effects seen on recollection were significantly reduced under incidental, as compared with intentional, encoding conditions, whereas the effects on familiarity were not modulated by the type of encoding.

The type of encoding did not have a large effect on the overall level of recollection, but it did influence famil- 


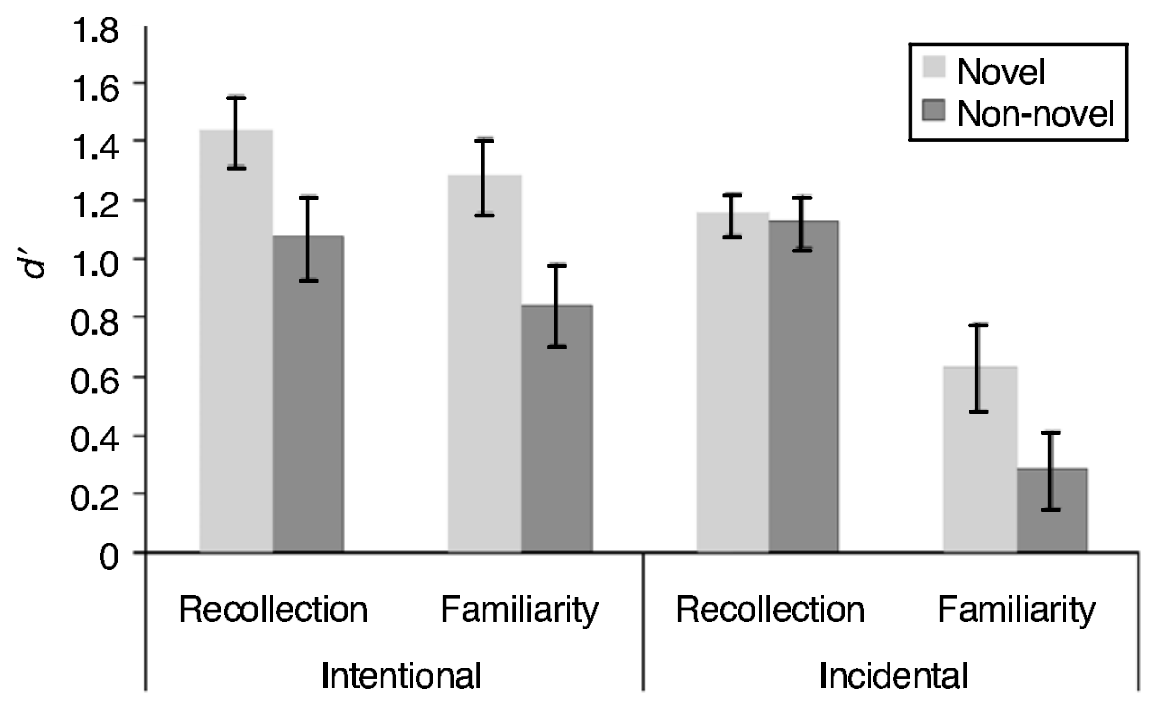

Figure 2. Experiment 2: Estimates of recollection and familiarity for the intentional and the incidental encoding conditions.

iarity. Although no a priori predictions were made about this main effect, the results suggest that the intention to encode, at least with the present materials and encoding instructions, benefits familiarity but does not benefit recollection. Note, however, that Lampinen, Copeland, and Neuschatz (2001) also examined the effects of intention on remember and know responses. When those responses are used to estimate recollection and familiarity, the intention to retrieve is found to increase both recollection and familiarity.

\section{GENERAL DISCUSSION}

The influence of stimulus novelty on recognition memory was examined using a von Restorff (1933) paradigm in which items were made novel by presenting them in a distinctive color. The results are consonant with a growing body of literature in which it is indicated that novel or distinctive items lead to better recognition memory performance than do non-novel items (Fabiani \& Donchin, 1995; Kinsbourne \& George, 1974; Parker et al., 1998; Tulving \& Kroll, 1995). The present results, however, extend those findings by showing that both recollection and familiarity-based recognition responses can be influenced by novelty. Moreover, in agreement with results from tests of free recall (e.g., Gleitman \& Gillett, 1957; Postman \& Phillips, 1954; Saltzman \& Carterette, 1959), the novelty effects on recollection were reduced under incidental, as compared with intentional, encoding conditions. In contrast, the novelty effects on familiarity were not influenced by the encoding manipulation. To the extent that the novelty effects on recollection, but not on familiarity, were sensitive to the type of encoding, the results suggest that there are a least two partially separable effects of novelty on recognition memory.

The von Restorff effects in the present study are generally consistent with a previous study (Rajaram, 1998) in which the effects of orthographic distinctiveness were examined. In that study, orthographically distinctive words were found to lead to more remember responses (.61) than did common words (.49), which is consistent with the present results, whereas both types of words led to the same proportion of know responses (.29). The fact that know responses did not change might suggest that familiarity was not influenced by distinctiveness, which is not consistent with the present findings. However, know responses do not provide an index of familiarity, because they are mathematically constrained by recollection. That is, subjects are not instructed to respond know whenever an item is familiar; rather, they are instructed to respond know whenever it is familiar and not recollected. If one examines the probability of a know response given that the subjects had the opportunity to make a know response (i.e., it was not recollected), the results show that familiarity was influenced by orthographic distinctiveness. That is, when familiarity is estimated as the probability of a know response given that the item was not recollected [i.e., $F=K /(1-R)$ ], estimates of familiarity are found to increase from .57 to .73 for the distinct items in that experiment. Thus, as with the von Restorff effects in the present experiments, orthographic distinctiveness was found to increase both recollection and familiarity. Note that false alarms differed for the distinct and the nondistinct items in Rajaram's (1998) experiment, but even when false alarms are subtracted from hits, the pattern of results is unchanged.

The present results are only partially consistent with the results from the previous study of von Restorff effects in nonhuman primates (Parker et al., 1998). That study indicated that lesions that included the parahippocampal gyrus, which has been implicated in familiaritybased recognition, eliminated the novelty effect. These results are thus consistent with the present findings in suggesting that the familiarity process plays a critical 
role in the von Restorff effect. However, that study also indicated that lesions designed to disrupt the function of the hippocampus, a region implicated in recollectionbased recognition, did not influence the novelty effect, suggesting that recollection was not involved in the novelty effect. These results conflict with the present findings, in which recollection was found to be sensitive to novelty. The reason for this discrepancy is not known. Whether it reflects a difference in how human and nonhuman primates complete recognition tests or whether it has to do with the particular experimental procedures used in the two studies is not clear. For example, given that we found that the effects of novelty on recollection depended on the manner in which items were encoded, differences in encoding strategies across species may have played a critical role. Moreover, the study list in the present experiments was quite long, whereas in Parker et al.'s experiment a repeated study-test procedure was used in which the length of the study list varied for each animal, depending on its performance, and list lengths could be as short as two items. Further studies directly contrasting novelty effects in human and nonhuman primates will be necessary to fully address this issue.

\section{The Functional Nature of \\ Recollection and Familiarity}

Previous studies have indicated that recollection and familiarity differ in a number of ways, including retrieval speed and sensitivity to elaborative encoding (for a review, see Yonelinas, 2002). One additional difference that was entertained in the present study was that the two processes might differ in the sense that one process is more sensitive than the other to stimulus novelty. The present results show that both processes are sensitive to novelty, and thus the results indicate that sensitivity to novelty does not provide a simple way of differentiating these two types of recognition memory.

The present results clearly do not support the recollection/ distinctiveness hypothesis, in which recollection is expected to be particularly sensitive to stimulus novelty (e.g., Rajaram, 1998). In fact, unlike for recollection, pronounced effects of novelty on familiarity were found under both intentional and incidental encoding conditions. Nonetheless, could the subjective experience of familiarity in the absence of recollection (i.e., knowing) be roughly comparable for novel and non-novel items? In Rajaram's (1998) study, the probability of responding know to an old item did not change for orthographically distinct, as compared with orthographically common, words. Moreover, even when the accuracy of knowing was measured by subtracting false alarms from hits, performance was not influenced by orthographic distinctiveness. The results of the present experiments indicate that the proportions of know responses to the old items were similar for novel and non-novel items (averaged across experiments, the proportions of know responses for the novel and the non-novel items were .25 and .26 , respectively). However, the proportion of corrected know responses (hits minus false alarms) was greater for the novel than for the non-novel items. The average proportions of corrected know responses were .11 and .02 for novel and non-novel items, respectively, and this pattern was observed in each of the experiments. These results suggest that reports of knowing, like reports of remembering, can be influenced by stimulus novelty.

The present results are also inconsistent with a strong version of the familiarity/novelty hypothesis. Although familiarity was sensitive to novelty, recollection also increased for novel items, at least under intentional encoding conditions. One of the motivations behind the expectation that familiarity and novelty should be related was the speculation that the same process as that involved in detecting novelty might also be involved in familiarity-based recognition judgments. The present results indicate that the von Restorff effect cannot be explained solely as a familiarity-driven phenomenon. However, to the extent that familiarity was sensitive to novelty, the present results do leave open the possibility that there is some relation between the novelty response and familiarity-based recognition.

The present results indicate that the effects of novelty on recollection and familiarity were functionally distinct, suggesting that the mechanisms that produce novelty effects on these two processes may be different. Exactly what those mechanisms are is not yet clear. However, one speculation is that the effects of novelty on recollection may reflect enhanced encoding of novel items, whereas the effects on familiarity may reflect reduced interference at the time of retrieval (for discussions of encoding and retrieval accounts of the von Restorff effect, see Fabiani \& Donchin, 1995; Hunt \& Lamb, 2001; Karis, Fabiani, \& Donchin, 1984; Schmidt, 1991). Evidence that the effects of novelty on recollection were encoding related comes from the observation that the effect was modulated by the encoding instructions. Evidence that the effects of novelty on familiarity were retrieval related is provided by the finding that the encoding instructions did not influence the effect. Moreover, an examination of the proportion of know responses (see Table 1) indicates that novelty had a large effect on know responses to new items. That is, the new test items that were in the same color as the novel items were associated with lower false know rates than were the new items that were in the non-novel color. Given that the new items were not studied, it would seem that the novelty effect might be due, at least in part, to retrieval effects.

Another possible account of the difference in the effects of novelty on recollection and familiarity is that there may be both automatic and controlled responses to novel items during encoding and these two responses to novelty may differentially influence recollection and familiarity. That is, novel items may lead to an automatic orienting response (Knight, 1996; Knight \& Nakada, 1998; Schmidt, 1991) that may preferentially enhance subsequent familiarity-based recognition. In addition, novel items may also lead to further elaborative or controlled processing (e.g., Karis et al., 1984; Schmidt, 1991), 
and this elaborative processing may preferentially benefit recollection. Consistent with this possibility is the observation that novelty effects on recollection, but not on familiarity, were reduced by incidental encoding, a factor known to have differential effects on automatic and controlled processes (e.g., Hasher \& Zacks, 1979; Schneider \& Shiffrin, 1977).

To our knowledge, the present study is the first to demonstrate that both recollection and familiarity can be influenced by stimulus novelty. Although recollection and familiarity have been found to differ in a number of ways, the present results suggest that sensitivity to novelty does not provide a particularly simple way of differentiating between the two processes. Nonetheless, the conditions under which these processes were sensitive to novelty did differ; familiarity exhibited novelty effects under intentional and incidental encoding conditions, whereas recollection exhibited novelty effects only under intentional encoding conditions. These results suggest that there are two partially distinct responses to novelty, as measured in recognition memory.

\section{REFERENCES}

Aggleton, J. P., \& Brown, M. B. (1999). Episodic memory, amnesia and the hippocampal-anterior thalamic axis. Behavioral \& Brain Sciences, 22, 425-489.

Cimbalo, R. S. (1978). Making something stand out: The isolation effect in memory performance. In M. M. Grunneberg, P. E. Morris, \& R. N. Sykes (Eds.), Practical aspects of memory (pp. 101-110). New York: Academic Press.

Cimbalo, R. S., Capria, R. A., Neider, L. L., \& Wilkins, M. C. (1977). Isolation effect: Overall list facilitation in short-term memory. Acta Psychologica, 41, 419-432.

Eichenbaum, H., Otto, T., \& Cohen, N. J. (1994). Two functional components of the hippocampal memory system. Behavioral \& Brain Sciences, 17, 449-518.

FABIANi, M., \& Donchin, E. (1995). Encoding processes and memory organization: A model of the von Restorff effect. Journal of Experimental Psychology: Learning, Memory, \& Cognition, 21, 224-240.

GARDINER, J. M. (1988). Functional aspects of recollective experience. Memory \& Cognition, 16, 309-313.

GARDINER, J. M., \& JAVA, R. I. (1991). Forgetting in recognition memory with and without recollective experience. Memory \& Cognition, 19, 617-623.

Gardiner, J. M., \& Parkin, A. J. (1990). Attention and recollective experience in recognition memory. Memory \& Cognition, 18, 579-583.

Gleitman, H., \& Gillett, E. (1957). The effect of intention upon learning. Journal of General Psychology, 57, 137-149.

HASHER, L., \& ZACKS, R. T. (1979). Automatic and effortful processes in memory. Journal of Experimental Psychology: General, 108, 356388.

Hunt, R. R. (1995). The subtlety of distinctiveness: What von Restorff really did. Psychonomic Bulletin \& Review, 2, 105-112.

Hunt, R. R, \& LAmb, C. A. (2001). What causes the isolation effect? Journal of Experimental Psychology: Learning, Memory, \& Cognition, 27, 1359-1366.

IsHIHARA, S. (1998). The series of plates designed as a test for colour deficiency. Tokyo: Kanehara \& Co.

KARIS, D., FABIANI, M., \& Donchin, E. (1984). "P300" and memory: Individual differences in the von Restorff effect. Cognitive Psychology, 16, 177-216.

Kinsbourne, M., \& George, J. (1974). The mechanism of the wordfrequency effect on recognition memory. Journal of Verbal Learning \& Verbal Behavior, 13, 63-69.
KNIGHT, R. T. (1996). Contribution of the human hippocampal region to novelty detection. Nature, $\mathbf{3 8 3}, 256-259$.

Knight, R. T., \& NAKadA, T. (1998). Cortico-limbic circuits and novelty: A review of EEG and blood flow data. Reviews in the Neurosciences, 9, 57-70.

KoyANAGI, K. (1957). Studies in incidental learning: II. Interserial interference. Tohoku Psychologica Folia, 15, 1-12.

Lampinen, J. M., Copeland, S. M., \& Neuschatz, J. S. (2001). Recollections of things schematic: Room schemas revisited. Journal of Experimental Psychology: Learning, Memory, \& Cognition, 27, 1211-1222.

MacMillan, N. A., \& Creelman, C. D. (1991). Detection theory: A user's guide. New York: Cambridge University Press.

MANDLER, G. (1980). Recognizing: The judgment of previous occurrence. Psychological Review, 87, 252-271.

MasterClips [Computer software] (1998). San Rafael, CA: IMSI.

Parker, A., Wilding, E., \& Ackerman, C. (1998). The von Restorff effect in visual object recognition memory in humans and monkeys: The role of frontal/perirhinal interaction. Journal of Cognitive Neuroscience, 10, 691-703.

Postman, L., \& Phillips, L. W. (1954). Studies in incidental learning: I. The effect of crowding and isolation. Journal of Experimental Psychology, 48, 48-56.

Rajaram, S. (1996). Perceptual effects on remembering: Recollective processes in picture recognition memory. Journal of Experimental Psychology: Learning, Memory, \& Cognition, 22, 365-377.

RAJARAM, S. (1998). The effects of conceptual salience and perceptual distinctiveness on conscious recollection. Psychonomic Bulletin \& Review, 5, 71-78.

Saltz, E., \& Newman, S. E. (1959). The von Restorff isolation effect: Test of the intralist association assumption. Journal of Experimental Psychology, 58, 445-451.

Saltzman, I. J., \& Carterette, T. (1959). Incidental and intentional learning of isolated and crowded items. American Journal of Psychology, 72, 230-235.

SchmidT, S. R. (1991). Can we have a distinctive theory of memory? Memory \& Cognition, 19, 523-542.

Schneider, W., \& Shiffrin, R. M. (1977). Controlled and automatic human information processing: I. Detection, search, and attention. Psychological Review, 84, 1-66.

SoKolov, E. N. (1963). Higher nervous functions: The orienting reflex. Annual Review of Physiology, 25, 545-580.

Tulving, E. (1985). Memory and consciousness. Canadian Psychologist, 26, 1-12.

Tulving, E., \& Kroll, N. (1995). Novelty assessment in the brain and long-term memory encoding. Psychonomic Bulletin \& Review, 2, 387-390.

von RESTORFF, H. (1933). Über die Wirkung von Bereichsbildungen im Spurenfeld. Psychologische Forschung, 18, 299-342.

Wallace, W. P. (1965). Review of the historical, empirical, and theoretical status of the von Restorff phenomenon. Psychological Bulletin, 63, 410-424.

Yonelinas, A. P. (2001). Consciousness, control, and confidence: The 3 Cs of recognition memory. Journal of Experimental Psychology: General, 130, 361-379.

Yonelinas, A. P. (2002). The nature of recollection and familiarity: A review of 30 years of research. Journal of Memory \& Language, 46, 441-517.

Yonelinas, A. P., \& JACOBY, L. L. (1995). The relation between remembering and knowing as bases for recognition: Effects of size congruency. Journal of Memory \& Language, 34, 622-643.

Yonelinas, A. P., Kroll, N. E. A., Quamme, J. R., Lazzara, M. M., Sauve, M. J., Widaman, K. F., \& KNight, R. T. (2002). Effects of extensive temporal lobe damage or mild hypoxia on recollection and familiarity. Nature Neuroscience, 5, 1236-1241.

(Manuscript received October 29, 2002; revision accepted for publication June 9, 2003.) 